\title{
Conditions of progress in mathematics teacher education
}

\author{
João Pedro da Ponte
}

Published online: 25 September 2009

(C) Springer Science+Business Media B.V. 2009

The progress of mathematics teacher education as a field of study depends on theoretical and empirical work. Both kinds of work are intimately related. Any scientific article that reports an empirical study needs to be based in theoretical constructs, and, at the very end, should contribute to a better understanding of these concepts or, at least, of their power for understanding specific problems, and any theoretical work, to make sense, requires some sort of empirical application.

The articles in this issue of JMTE draw on several key theoretical concepts in mathematics teacher education: content knowledge and pedagogical content knowledge (Davis), teachers' beliefs (Cross, Davis), teachers' practices (Cross, Ryken), and teacher learning (Ryken). As a subfield of the social sciences, we cannot expect that all the authors share the same view about such notions. However, we must be able to understand how they conceptualize their major theoretical concepts, relate them to each other, note their similarities and differences, and regard the implications of the different choices.

Teachers' knowledge of mathematics includes teacher knowledge of mathematical facts and representations, concepts, procedures, and other information, as well as the ability to solve problems, to construct and use models, to establish connections within and outside mathematics, to reason, to prove, and to evaluate results. In this issue, Davis, in his article titled "Understanding the influence of two mathematics textbooks on prospective secondary teachers' knowledge" draws on the distinction between factual knowledge and conceptual knowledge that points toward different levels of elaboration. Other dimensions of knowledge in action, which are captured by the notions of problem solving, modeling, proving, and evaluating, are at the core of the current mathematics curriculum orientations for students' learning and also need the attention of mathematics teacher education researchers.

Teachers' pedagogical content knowledge blends "pedagogy" and "content" in a special way. Davis stresses two aspects, the educative representations that include "the most powerful analogies, illustrations, examples, explanations, and demonstrations", and

\section{J. P. da Ponte ( $\bowtie)$}

Instituto de Educação, Universidade de Lisboa, Edifício C6, Campo Grande, 1749-016 Lisbon,

Portugal

e-mail: jpponte@ie.ul.pt 
topic perceptivity, "an understanding of what makes the learning of specific topics easy or difficult." Pedagogical content knowledge is an appealing notion, as it resonates with the experience and concerns of teachers and teacher educators for whom both content and pedagogy are important elements. This notion has proved to be very fruitful in research with teachers. However, it is also a notion that has been reframed over and over again, both regarding its content and its nature: Is it declarative and formal knowledge, that can be learned and assessed using verbal language, or is it essentially practical and implicit knowledge that has to appraised and developed through practice? Pedagogical content knowledge is the knowledge of experienced teachers, seen by their colleagues as professional leaders, or is it the knowledge that researchers posit teachers must have?

Teachers' beliefs is another key notion that frames much research on teachers. Beliefs are closely related to knowledge, establishing a bridge between the cognitive and the affective domain. For some researchers, beliefs are a special kind of knowledge; for others, they have different epistemic status. In this issue, Cross, in the article titled "Alignment, cohesion and change: Examining mathematics teachers' belief structures and their influence on instructional practice," sees beliefs, as "embodied conscious and unconscious ideas and thoughts about oneself, the world, and one's position in it, developed through membership in various social groups", and gives special attention to teachers' "mathematics beliefs" - beliefs about the nature of mathematics, about mathematics teaching, and about student learning. Davis also refers to beliefs about mathematics and mathematics teaching. There is little doubt that beliefs are a useful concept to describe teachers' activity. However, we are still in need of a better understanding about how beliefs develop, change, and what factors mediate their influence on practice.

The notion of teachers' practices is another central construct. Often, practices are regarded as "actions", "acts", "behaviours," or "gestures" that can be observed and categorized in an objective way. However, teachers may be doing similar actions with quite different purposes in rather different activity systems. An element of stability and recurrence in a given context is essential in the notion of practice. In addition, there is a reflexive relationship between individual activities and social practices - the activities of the individual are constitutive of social practices and, at the same time, social practices give form and meaning to the activities of the individual. In this issue, Cross looks at instructional practices, with special attention to organizing the classroom environment, the classroom discourse, and interactions promoted, and the types and use of assessments. Ryken also looks at assessment practices.

Finally, another key notion is that of teacher learning or teacher professional development. Teachers learn about mathematics, curriculum frameworks and management, ways of teaching mathematics, classroom materials, handling classroom discourse, students' strategies and difficulties, assessment instruments procedures, and implications, institutional, social, and professional values, opportunities, and constrains. Teachers develop professionally as they become more able to use their resources to solve the professional problems that they face in their daily activity. Through these processes they develop their professional identity as teachers, another notion hinted at by Ryken, in the article titled "Multiple representations as sites for teacher reflection about mathematics learning". As this author indicates, teacher learning has been analyzed from both a cognitive perspective, putting emphasis in developing concepts and problem solving strategies, and a socialization perspective, balancing choices, and constraints within institutional cultures. Both perspectives address different issues and shed light on different phenomena. How can we connect them in a meaningful and productive ways? What price do we pay in making such connection? 
Looking at other issues of this Journal, we could expand this list. The fact that we have a handful of major concepts in mathematics teacher education is an important sign of progress. However, such concepts will only be useful if we take care to discuss and clarify the meaning we give to them, and if we establish connections and relations among them, which help a better understanding of the ways teachers work, think, and learn at the different stages of their careers and institutional working conditions. 